\title{
Lower bounds on the estimation performance in low complexity quantize-and-forward cooperative systems
}

\author{
lancu Avram* Nico Aerts and Marc Moeneclaey
}

\begin{abstract}
Cooperative communication can effectively mitigate the effects of multipath propagation fading by using relay channels to provide spatial diversity. A relaying scheme suitable for half-duplex devices is the quantize-and-forward (QF) protocol, in which the information received from the source is quantized at the relay before being forwarded to the destination. In this contribution, the Cramer-Rao bound (CRB) is obtained for the case where all channel parameters in a QF system are estimated at the destination. The CRB is a lower bound (LB) on the mean square estimation error (MSEE) of an unbiased estimate and can thus be used to benchmark practical estimation algorithms. Additionally, the modified Cramer-Rao bound (MCRB) is also presented, which is a looser but computationally less complex bound. An importance sampling technique is developed to speed up the computation of the MCRBs, and the MSEE performance of a practical estimation algorithm is compared with the (M)CRBs. We point out that the parameters of the source-destination and relay-destination channels can be accurately estimated but that inevitably the source-relay channel estimate is poor when the instantaneous SNR on the relay-destination channel is low; however, in this case, the decoder performance is not affected by the inaccurate source-relay channel estimate.
\end{abstract}

Keywords: Cooperative communication; Estimation; Cramer-Rao bound; Modified Cramer-Rao bound

\section{Introduction}

In wireless communication, multipath signal propagation can cause destructive interference at the receiving antenna, giving rise to fading and limiting the maximum throughput of the system $[1,2]$. This phenomenon can be combatted by introducing diversity into the system, such as frequency diversity provided by orthogonal frequencydivision multiplexing (OFDM) [3] or spacial diversity provided by multiple-input multiple-output (MIMO) systems $[4,5]$. A novel means of providing diversity is cooperative communication, in which relays provide spatial diversity by creating multiple signal paths between the source and the destination [6]. Cooperative communication systems take advantage of the broadcast nature of the wireless medium. The information broadcast by the source terminal is received not only by the destination but also by other terminals nearby. Instead of ignoring this signal as is the

*Correspondence: iancu.avram@telin.ugent.be

Department of Telecommunications and Information Processing (TELIN, UGent), St-Pietersnieuwstraat 41, 9000 Ghent, BE, Belgium case in a classical communication system, in a cooperative system, these nearby terminals act as relays, forwarding to the destination the information received from the source and thus creating additional signal paths $[7,8]$.

Different strategies can be used to implement the information forwarding, including amplify-and-forward (AF) [9], quantize-and-forward (QF) [10], decode-and-forward (DF) [11], and coded cooperation [12]. The AF protocol, in which the relay amplifies the signal received from the source before sending it to the destination, is well known for its seemingly low-complexity implementation. However, when using half-duplex terminals which cannot send and receive data simultaneously, the information received from the source needs to be stored with high precision at the relay awaiting retransmission to the destination, requiring a large memory at the relay terminal. While this memory requirement might not be a concern for high-end mobile terminals, it can be of importance in low complexity applications such as sensor networks, where the sensor hardware complexity should be kept low in order to minimize production cost and to maximize battery life

\section{至 Springer}

C 2015 Avram et al. Open Access This article is distributed under the terms of the Creative Commons Attribution 4.0 International License (http://creativecommons.org/licenses/by/4.0/), which permits unrestricted use, distribution, and reproduction in any medium, provided you give appropriate credit to the original author(s) and the source, provide a link to the Creative Commons license, and indicate if changes were made. 
[13]. In order to meet these complexity demands, the QF protocol has been introduced. In the QF protocol, the data received from the source is coarsely quantized at the relay before being stored into memory, requiring significantly less memory space as compared to the AF protocol. Despite its low complexity, it has been shown that the QF protocol can achieve a very satisfactory error performance that is very close to that of AF $[10,14]$.

The majority of the research on cooperative systems has been performed under the assumption of perfect channel state information (CSI). While this is useful for obtaining various information-theoretical results, the real-life situation of imperfect CSI presents new challenges that need to be tackled. In contrast to a non-cooperative communication system where only the source-destination channel needs to be estimated, cooperative communication systems must also acquire the state of the source-relay and the relay-destination channels. Channel parameter estimation for QF has been discussed in [14, 15] for a protocol in which the relay estimates the source-relay (SR) channel and forwards its estimate to the destination, while [16] describes a QF system in which all channel parameters are estimated at the destination. The latter system benefits from the decreased relay-side complexity. However, it poses a more complex estimation problem, as the sourcerelay channel is not directly observed by the destination. In order to tackle this estimation problem, in [16], the source-relay channel is abstracted to be a discrete channel, characterized by a finite set of transition probabilities. In doing so, the estimation of the source-relay channel is greatly simplified, and the computational complexity at the destination is reduced. Furthermore, this makes the estimation of the estimation of the source-relay channel transition probabilities independent of the channel model and quantization method, making the results applicable to a large variety of systems.

Once an estimation algorithm has been obtained for a certain estimation problem, different approaches exist to benchmark the performance of the considered algorithm. A first approach is to compare the decoding error rate of a system in which the channel parameters are estimated to that of a system in which the channel parameters are considered to be known. The difference between the two error rates provides an indication about the performance of the estimation algorithm. A more fundamental way of studying the performance of an estimation algorithm is provided by the Cramer-Rao bound (CRB), which is a lower bound (LB) on the error variance of an unbiased estimate [17]; when an estimate exhibits an error variance that is close to the CRB, there is little room to improve the accuracy of the considered estimate. The opposite holds for an estimate having an error variance that is much higher as compared to the CRB. The CRB for the estimation of the channel parameters in a one-way
AF cooperative system using flat-fading channels has been obtained in [18], while in [19], the CRB is obtained for an $\mathrm{AF}$ and DF system in which multiple frequency offsets are considered. Lower bounds for the two-way relaying channel, in which two terminals aided by a relay communicate with one another, have been obtained in [20-23] for the AF protocol.

In this contribution at hand, the $(\mathrm{CRB})$ is obtained for a QF system in which all relevant channel parameters are jointly estimated at the destination. It is identified which channels are the most difficult to accurately estimate and to which degree the different channel estimates are coupled. By interpreting the cascade of the source-relay channel and the quantization operation as a single discreteinput discrete-output channel, the results obtained in this contribution can be used to benchmark a wide variety of cases with minimal adjustments, such as a multi-hop QF relaying scheme often found in sensor networks. Because the CRB is difficult to evaluate in some cases, the modified Cramer-Rao bound (MCRB) is also considered, which has a lower complexity compared to the CRB and converges to the latter at high SNR $[24,25]$. The MCRB for a similar system has been obtained in [26]. However, because the MCRB is not a tight bound at low SNR values, obtaining the value of the CRB is important in order to be able to benchmark the estimation performance over the complete SNR range. Moreover, the comparison between the CRB and MCRB also provides valuable insights.

The remainder of this contribution is organized as follows. In Section 2, the system model is introduced. In Section 3, an expression is obtained for the CRB, whereafter the MCRB is derived in Section 4. Next, in Section 5, the use of importance sampling (IS) is outlined, a technique that can be used to drastically shorten numerical simulation times [28], without affecting the accuracy of the obtained bounds. Finally, numerical results are presented in Section 6, whereafter conclusions are drawn in Section 7.

\section{Channel model}

In this contribution, a cooperative network is analyzed consisting of a direct path and a one-hop relaying channel, as depicted in Fig. 1. The relay is considered to be a halfduplex device, meaning that it cannot send and receive information simultaneously. In a first frame, the source broadcasts a sequence of $K M_{1}$-PSK symbols, denoted $\boldsymbol{c}_{s}$, which are received by both the relay and the destination. The relay quantizes the angle of the received samples using $\log _{2}\left(M_{2}\right)$ bits. These quantized samples, represented by a sequence of $K M_{2}$-PSK symbols denoted $\boldsymbol{c}_{r}$, are then forwarded to the destination in a second frame. At the destination, maximum-ratio combining (MRC) is used to combine the signals received from the source and relay. 


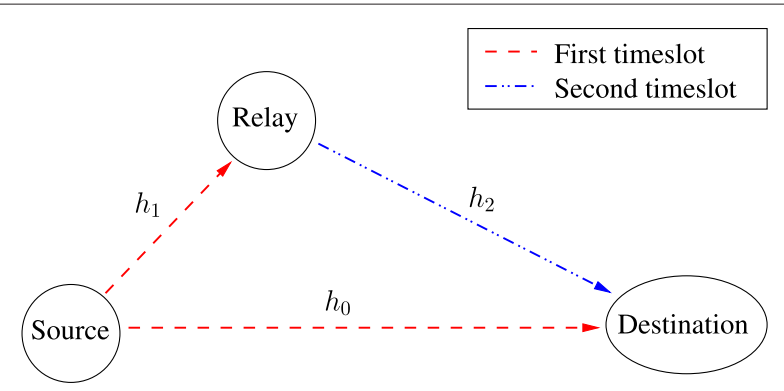

Fig. 1 A relay channel consisting of half-duplex devices

\subsection{Source-destination and relay-destination channels}

The source-destination (SD) and relay-destination (RD) channels are modeled as flat Rayleigh fading channels with additive white Gaussian noise. The SD and RD channel coefficients are denoted $h_{0}$ and $h_{2}$, respectively. The signals received by the destination from the source and the relay, denoted $\boldsymbol{r}_{0}$ and $\boldsymbol{r}_{2}$, respectively, are equal to

$$
\begin{aligned}
& \boldsymbol{r}_{0}=\sqrt{E_{s}} \boldsymbol{c}_{s} h_{0}+\boldsymbol{n}_{0} \\
& \boldsymbol{r}_{2}=\sqrt{E_{r}} \boldsymbol{c}_{r} h_{2}+\boldsymbol{n}_{2} .
\end{aligned}
$$

Assuming the normalization $\left|\mathbf{c}_{s}\right|^{2}=\left|\mathbf{c}_{r}\right|^{2}=K$, the quantities $E_{s}$ and $E_{r}$ denote the transmitted energy per symbol at the source and the relay, respectively. The channel coefficients $h_{0}$ and $h_{2}$ are considered to be constant during a frame and have a zero mean circular symmetric complex gaussian (ZMCSCG) distribution with variances $N_{h_{0}}=1 / d_{0}{ }^{n_{\text {loss }}}$ and $N_{h_{2}}=1 / d_{2}{ }^{n_{\text {loss }}}$, respectively. The quantities $d_{0}$ and $d_{2}$ represent the distance between the source and the destination and between the relay and the destination, while $n_{\text {loss }}$ denotes the path loss exponent. The components of the noise vectors $\boldsymbol{n}_{0}$ and $\boldsymbol{n}_{2}$ are also ZMCSCG distributed with respective variances $N_{0}$ and $N_{2}$.

\subsection{Equivalent source-relay channel}

Due to the quantization operation, both the source and the relay transmit discrete symbols from a PSK constellation. Hence, the cascade of the source-relay (SR) channel and the quantization operation at the relay can be interpreted as an equivalent discrete-input discrete-output communication channel with $M_{1}$ input values and $M_{2}$ output values. Assuming that the actual SR channel and the quantizer are memoryless and time-invariant over a frame, this equivalent SR channel is fully characterized by $M_{2} \times M_{1}$ transition probabilities $\pi_{q, m}$, which determine the probability of a symbol sent by the relay conditioned on the symbol sent by the source in the corresponding symbol interval, i.e., for $q=0, \cdots, M_{2}-1$ and $m=$ $0, \cdots, M_{1}-1$,

$$
\pi_{q, m}(k)=P\left[c_{r}(k)=\chi_{M_{2}}(q) \mid c_{s}(k)=\chi_{M_{1}}(m)\right],
$$

with $\chi_{M}(x)=e^{\frac{j 2 \pi x}{M}}$. Denoting by $r_{1}(k)$ the $k$ th sample at the input of the relay, the transition probabilities $\pi_{q, m}$ can be computed from the SR channel likelihoods $p\left(r_{1}(k) \mid c_{s}(k)=\chi_{M_{1}}(m)\right)$ and the quantization rule that maps $r_{1}(k)$ to $\chi_{M_{2}}(q)$. Results in this paper will be derived in terms of the transition probabilities, without specifying the underlying SR channel likelihood and quantization rule.

It is further assumed that $M_{2} / M_{1}$ is integer and that the quantization operation exhibits circular symmetry with respect to the symbols sent by the source, so that

$$
\pi_{q+\frac{M_{2}}{M_{1}} m, m}=\pi_{q, 0}=\tau_{q} .
$$

Hence, the equivalent SR channel is characterized by the transition probabilities $\left\{\tau_{q}, q=0, \cdots, M_{2}-1\right\}$.

\section{The Cramer-Rao bound}

The CRB is a lower bound (LB) on the mean square error (MSE) of an unbiased estimate. In the current system model, the SD and RD channel coefficients, $h_{0}$ and $h_{2}$, as well as the SR transition probabilities $\tau_{q}$ are unknown and need to be estimated before the information transmitted by the source can be reliably detected at the destination. In order to keep the complexity of the relay terminal low, it is imposed that all parameters are estimated at the destination. It is further assumed that the destination does not posses any a priori information on the different channel parameters.

The unknown channel parameters are grouped into the real-valued vector $\boldsymbol{\theta}$, which is given by

$$
\boldsymbol{\theta}=\left(\mathfrak{R}\left(h_{0}\right), \mathfrak{I}\left(h_{0}\right), \boldsymbol{\tau}^{T}, \mathfrak{R}\left(h_{2}\right), \mathfrak{I}\left(h_{2}\right)\right)^{T},
$$

with $\tau=\left(\tau_{0}, \tau_{1}, \cdots, \tau_{M_{2}-2}\right)^{T}$. Note that $\tau_{M_{2}-1}$ is not contained in $\boldsymbol{\theta}$, because it is not an independent parameter $\left(\tau_{M_{2}-1}=1-\tau_{0}-\ldots-\tau_{M_{2}-2}\right)$. In order to obtain the CRB, the Fisher information matrix (FIM) $\boldsymbol{J}(\boldsymbol{\theta})$ is introduced, the elements of which are defined as [17]

$$
\boldsymbol{J}(\boldsymbol{\theta})_{i, j}=\mathbb{E}_{\boldsymbol{r}}\left[\frac{\partial}{\partial \theta_{i}} \ln p(\boldsymbol{r} ; \boldsymbol{\theta}) \frac{\partial}{\partial \theta_{j}} \ln p(\boldsymbol{r} ; \boldsymbol{\theta})\right],
$$

with $\boldsymbol{r}=\left(\boldsymbol{r}_{0}, \boldsymbol{r}_{2}\right)$. It is shown in Appendix A that the elements of the FIM can be expressed as

$$
\boldsymbol{J}(\boldsymbol{\theta})_{i, j}=\mathbb{E}_{\boldsymbol{r}}\left[X_{i}(\boldsymbol{r} ; \boldsymbol{\theta}) X_{j}(\boldsymbol{r} ; \boldsymbol{\theta})\right],
$$

where the expression for $X_{x}(\boldsymbol{r} ; \boldsymbol{\theta})$ is given in (23). In order to evaluate $X_{x}(\boldsymbol{r} ; \boldsymbol{\theta})$, the a posteriori probabilities $p\left(c_{s}(k) \mid \boldsymbol{r} ; \boldsymbol{\theta}\right)$ of the source symbols are needed. These depend on the structure of the channel code and are obtained at the destination using a soft decoder that operates on $\boldsymbol{r}$. Using the notation $\hat{x}$ to represent an unbiased estimate of $x$, it follows from [17] that 


$$
\mathbb{E}_{\boldsymbol{r}}\left[(\boldsymbol{\theta}-\hat{\boldsymbol{\theta}})(\boldsymbol{\theta}-\hat{\boldsymbol{\theta}})^{H}\right] \geq \boldsymbol{J}^{-1}(\boldsymbol{\theta}),
$$

where $\boldsymbol{A} \geq \boldsymbol{B}$ implies that $\boldsymbol{A}-\boldsymbol{B}$ is positive-semidefinite matrix. Using (5), a LB can be obtained on the mean square estimation error (MSEE) of the various channel parameters as is shown in the next subsections. Note that the value of this $L B$ depends on the specific realization of $\boldsymbol{\theta}$. In order to obtain a LB that is independent of $\boldsymbol{\theta}$, the obtained expressions need to be averaged over $\boldsymbol{\theta}$, as will be done numerically in Section 6 .

\subsection{SD and RD channel coefficients}

Once the elements of the FIM have been calculated, a LB on the MSEE of the SD and RD channel coefficients is obtained by noting that $h_{0}$ and $h_{2}$ can be expressed in terms of $\boldsymbol{\theta}$ as $h_{0}=\boldsymbol{u} \boldsymbol{\theta}$ and $h_{2}=\boldsymbol{w} \boldsymbol{\theta}$, with $\boldsymbol{u}=\left(1, j, \mathbf{0}_{M_{2}+1}\right)$ and $\boldsymbol{w}=\left(\mathbf{0}_{M_{2}+1}, 1, j\right)$, where $j^{2}=-1$. This yields the following $\mathrm{CRB}$ on the MSE arising from the estimation of $h_{0}$ :

$$
\mathbb{E}_{\boldsymbol{r}}\left[\left|h_{0}-\hat{h_{0}}\right|^{2}\right] \geq\left(\boldsymbol{u} \boldsymbol{J}^{-1}(\boldsymbol{\theta}) \boldsymbol{u}^{H}\right)=\mathrm{CRB}_{h_{0}}(\boldsymbol{\theta}) .
$$

The CRB on the MSE arising from the estimation of $h_{2}$ is obtained by substituting $\boldsymbol{u}$ for $\boldsymbol{w}$ in (6).

\subsection{SR transition probabilities}

Considering that $\boldsymbol{\tau}=\boldsymbol{V} \boldsymbol{\theta}$ with

$$
\boldsymbol{V}=\left[\begin{array}{lllll}
0 & 0 & 0 & 0 \\
0 & 0 & \mathbf{I}_{M_{2}-1} & 0 & 0 \\
0 & 0 & -1-1 & 0 & 0
\end{array}\right],
$$

the CRB on the MSE arising from the estimation of $\tau$ is given by

$$
\mathbb{E}_{\boldsymbol{r}}\left[(\boldsymbol{\tau}-\hat{\boldsymbol{\tau}})^{H}(\boldsymbol{\tau}-\hat{\boldsymbol{\tau}})\right] \geq \operatorname{Tr}\left(\boldsymbol{V} \boldsymbol{J}^{-1}(\boldsymbol{\theta}) \boldsymbol{V}^{H}\right)=\mathrm{CRB}_{\tau}(\boldsymbol{\theta}) .
$$

\subsection{Known parameter subset}

It will be useful to also consider the CRB related to the estimation of a parameter subset $\left\{\theta_{i}, i \in C\right\}$ under the assumption that the complementary parameter set $\left\{\theta_{j}, j \in\right.$ $\tilde{C}$ \} is known, in order to evaluate the impact, on the estimation of the former subset, of whether or not the latter subset is known. The corresponding FIM is obtained by removing from the original FIM the $j$ th rows and $j$ th columns that correspond to $j \in \tilde{C}$, leaving only the rows and columns that correspond to the unknown parameters $\left\{\theta_{i}, i \in C\right\}$.

\section{The modified Cramer-Rao bound}

As shown in Appendix A, the calculation of the elements of the FIM involves the computation of the a posteriori probabilities of the source symbols, making it difficult to obtain a closed-form expression for the CRB. Therefore, when using non-trivial channel codes, the CRB needs to be obtained using numerical simulations methods (see Section 5) that are quite time-consuming. In order to avoid the computational complexity associated with the CRB, we also consider the modified CRB (MCRB), which does not need the a posteriori probabilities of the source symbols (the MCRB assumes the source symbols are known by the receiver). The MCRB is a looser bound compared to the CRB; the CRB approaches the MCRB at high signal to noise ratios, where $p\left(c_{s}(k) \mid \boldsymbol{r}, \boldsymbol{\theta}\right) \approx 1$ for $c_{s}(k)$ equal to the symbol actually transmitted. Due to the less complex mathematics of the MCRB, simulation times are greatly reduced and in some cases closed-formed expressions can be found. The MCRB is obtained by substituting in (6) and (7) the inverse of the FIM with the inverse of the modified Fisher information matrix (MFIM), defined as [25]

$\boldsymbol{J}_{M}(\boldsymbol{\theta})_{i, j}=\mathbb{E}_{\boldsymbol{c}_{s}}\left[\mathbb{E}_{\boldsymbol{r} \mid \boldsymbol{c}_{s}}\left[\frac{\partial}{\partial \theta_{i}} \ln p\left(\boldsymbol{r} \mid \boldsymbol{c}_{s} ; \boldsymbol{\theta}\right) \frac{\partial}{\partial \theta_{j}} \ln p\left(\boldsymbol{r} \mid \boldsymbol{c}_{s} ; \boldsymbol{\theta}\right)\right]\right]$.

It follows from (2) that

$$
\begin{aligned}
p\left(r(k) \mid c_{s}(k)\right. & \left.=\chi_{M_{1}}(m) ; \boldsymbol{\theta}\right)\left.\right|_{r(k)=y} \\
& =\left.p\left(r(k) \mid c_{s}(k)=\chi_{M_{1}}(0) ; \boldsymbol{\theta}\right)\right|_{r(k)=y \chi_{M_{1}}^{*}(m)},
\end{aligned}
$$

so that in (8) the conditional expectation $\mathbb{E}_{\boldsymbol{r} \mid \boldsymbol{c}_{s}}$ [.] does not depend on the particular realization of $\boldsymbol{c}_{s}$. Hence, the outer expectation in (8) can be omitted, yielding

$$
\boldsymbol{J}_{M}(\boldsymbol{\theta})_{i, j}=\mathbb{E}_{\boldsymbol{r} \mid \boldsymbol{c}_{s}}\left[\frac{\partial}{\partial \theta_{i}} \ln p\left(\boldsymbol{r} \mid \boldsymbol{c}_{s} ; \boldsymbol{\theta}\right) \frac{\partial}{\partial \theta_{j}} \ln p\left(\boldsymbol{r} \mid \boldsymbol{c}_{s} ; \boldsymbol{\theta}\right)\right] .
$$

As shown in Appendix B, $\boldsymbol{J}_{M}$ is a block-diagonal matrix equal to

$$
\boldsymbol{J}_{M}=\left[\begin{array}{cc}
\boldsymbol{J}_{M, \mathrm{SD}} & 0 \\
0 & \boldsymbol{J}_{M, \mathrm{SRD}}
\end{array}\right]
$$

where $\boldsymbol{J}_{M, \mathrm{SD}}=\left(\frac{2 K E_{s}}{N_{0}}\right) \mathbf{I}_{2}$ and $\boldsymbol{J}_{M, \mathrm{SRD}}$ are the MFIMs related to the estimation of the SD channel parameters $\left\{\theta_{0}, \theta_{1}\right\}$ and the source-relay-destination (SRD) channel parameters $\overline{\boldsymbol{\theta}}=\left\{\theta_{i}, i>1\right\}$, respectively. It is shown in Appendix B that the elements of $\boldsymbol{J}_{M, \mathrm{SRD}}$ can be expressed as

$$
\boldsymbol{J}_{M, \mathrm{SRD}}(\overline{\boldsymbol{\theta}})_{i, j}=K \mathbb{E}_{r_{2} \mid c_{s}}\left[\bar{X}_{i}\left(r_{2}, c_{s} ; \overline{\boldsymbol{\theta}}\right) \bar{X}_{j}\left(r_{2}, c_{s} ; \overline{\boldsymbol{\theta}}\right)\right],
$$

where $\bar{X}_{i}\left(r_{2}, c_{s} ; \overline{\boldsymbol{\theta}}\right)$ is given by (35). The expression for the components of $\boldsymbol{J}_{M, \mathrm{SRD}}$ is given in (37) and (38). Because of the block-diagonal nature of $\boldsymbol{J}_{M}$, the SD channel parameters are decoupled from the SRD channel parameters: the MCRB corresponding to the SD (the SRD) channel parameters does not depend on whether or not the parameters of the SRD (the SD) channel are known. 


\subsection{SD channel}

Given the value of $\boldsymbol{J}_{M, \mathrm{SD}}$, the MCRB corresponding to the estimation of the SD channel coefficient $h_{0}$ becomes

$$
\mathbb{E}\left[\left|\hat{h_{0}}-h_{0}\right|^{2}\right] \geq \frac{N_{0}}{K E_{s}}=\mathrm{MCRB}_{h_{0}} .
$$

\subsection{SRD channel}

In order to obtain the MCRB for the parameters $\left(\tau, h_{2}\right)$ of the SRD channel, the expectation in (10) must be computed using Monte-Carlo (MC) methods (see Section 5). However, in a few special cases considered below, closedform expressions can be obtained.

\subsubsection{Perfect RD channel}

When the RD channel is perfect, the destination receives the message from the relay unaltered, i.e., $\boldsymbol{r}_{2}=\boldsymbol{c}_{r}$. In this case, the estimation of the SRD channel parameters reduces to the estimation of the transition probabilities of the equivalent SR channel. Introducing $\tilde{\boldsymbol{\theta}}=$ $\left(\tau_{0}, \tau_{1}, \ldots, \tau_{M_{2}-2}\right)^{T}$, the resulting FIM has dimension $\left(M_{2}-1\right) \times\left(M_{2}-1\right)$, and is equal to

$\boldsymbol{J}_{M, \mathrm{SR}}(\tilde{\boldsymbol{\theta}})_{i, j}=K \mathbb{E}_{c_{r} \mid c_{s}}\left[\frac{\partial}{\partial \tilde{\theta}_{i}} \ln p\left(c_{r} \mid c_{s} ; \tilde{\boldsymbol{\theta}}\right) \frac{\partial}{\partial \tilde{\theta}_{j}} \ln p\left(c_{r} \mid c_{s} ; \tilde{\boldsymbol{\theta}}\right)\right]$.

Using (37), (11) can be written as

$$
\boldsymbol{J}_{M, \mathrm{SR}}(\tilde{\boldsymbol{\theta}})_{i, j}=K\left(\frac{\delta_{i-j}}{\tau_{i}}+\frac{1}{\tau_{M_{2}-1}}\right),
$$

where $\delta_{x}$ denotes the Kronecker delta function. Hence, the MFIM can be represented as:

$$
\boldsymbol{J}_{M, \mathrm{SR}}(\tilde{\boldsymbol{\theta}})=K\left(D^{-1}+\tau_{M_{2}-1}^{-1} \mathbf{1}_{M_{2}-1}^{T} \mathbf{1}_{M_{2}-1}\right),
$$

with $\boldsymbol{D}$ a diagonal matrix with diagonal elements equal to $\left(\tau_{0}, \tau_{1}, \ldots, \tau_{M_{2}-2}\right)$. Using the Woodbury matrix identity [27] to calculate the inverse of the MFIM yields

$$
\boldsymbol{J}_{M, \mathrm{SR}}^{-1}(\tilde{\boldsymbol{\theta}})=\frac{1}{K}\left(\boldsymbol{D}-\tilde{\boldsymbol{\theta}}^{H} \tilde{\boldsymbol{\theta}}\right)
$$

The MCRB corresponding to the estimation of $\tau$ is obtained by substituting (13) into (7) with $\boldsymbol{V}=$ $\left(\mathbf{I}_{M_{2}-1},-\mathbf{1}_{M_{2}-1}^{T}\right)^{T}$, yielding

$$
\mathbb{E}\left[(\boldsymbol{\tau}-\hat{\boldsymbol{\tau}})^{H}(\boldsymbol{\tau}-\hat{\boldsymbol{\tau}})\right] \geq \frac{1}{K}\left(1-\sum_{q=1}^{M_{2}} \tau_{q}^{2}\right) .
$$

\subsubsection{Perfect equivalent SR channel}

When the equivalent SR channel is perfect, a symbol transmitted by the relay is completely determined by the corresponding symbol transmitted by the source; stated differently, all but one transition probabilities are zero, and the nonzero transition probability equals one. In this case, the estimation of the SRD parameters reduces to the estimation of the RD channel coefficient $h_{2}$. It can be shown that one obtains $\mathbf{J}_{M, \mathrm{RD}}=\left(\frac{2 K E_{r}}{N_{2}}\right) \mathbf{I}_{2}$, and subsequently

$$
\mathbb{E}\left[\left|\hat{h_{d}}-h_{d}\right|^{2}\right] \geq \frac{N_{2}}{K E_{r}} \text {. }
$$

\section{Importance sampling}

In general, the evaluation of the FIM (4) and the MFIM (9) involves expectations that cannot be obtained in closed form, so that we must resort to MC techniques. However, when some of the transition probabilities of the SR channel are very small, the computational effort required to obtain an accurate value for the (M)CRB can be very high. This can be understood by calculating the MCRB corresponding to the case of a perfect RD channel and using a MC approach instead of an analytical one. A straightforward MC approach to obtain $\boldsymbol{J}_{M, \mathrm{SR}}(\tilde{\boldsymbol{\theta}})_{i, j}$ involves the following approximation of (12):

$\boldsymbol{J}_{M, \mathrm{SR}}(\tilde{\boldsymbol{\theta}})_{i, j} \approx \frac{K}{N} \sum_{n=1}^{N}\left(\frac{I_{i}^{(n)} \delta_{i-j}}{\tau_{i}^{2}}+\frac{I_{M_{2}-1}^{(n)}}{\tau_{M_{2}-1}^{2}}\right) i, j=0,1, \ldots, M_{2}-2$,

where $I_{q}^{(n)}=1$ if $c_{r}^{(n)}=c_{s} e^{\frac{j 2 \pi q}{M_{2}}}$ and 0; otherwise, $c_{s}$ is an arbitrary symbol from the $M_{1}$-PSK constellation (say, $c_{s}=$ 1 ), and $\left\{c_{r}^{(n)}, n=1,2, \ldots, N\right\}$ is a sequence of i.i.d random variables, generated according to $\operatorname{Pr}\left[c_{r}^{(n)}=c_{s} e^{\frac{j 2 \pi q}{M_{2}}}\right]=$ $\tau_{q}, q=0,1, \ldots, M_{2}-1$. Suppose that for given $\mathrm{N}, \tau_{i 1}$ and $\tau_{i 2}$ are both much smaller than $1 / N$, so that it is likely to have $I_{i 1}^{(n)}=I_{i 2}^{(n)}=0$ for $n=1,2, \ldots, N$. In this case, the estimate (16) of the MFIM is singular (it has identical $i_{1}$ th and $i_{2}$ th rows), so that its inverse does not exist. Hence, to obtain meaningful results using (16), very large values of $\mathrm{N}$ (and, therefore, long simulation times) are required when some of the transition probabilities are very small.

These simulation times can significantly be shortened by the use of importance sampling (IS) [28]. For the example of the perfect RD channel, this involves generating $\left\{c_{r}^{(n)}, n=1,2, \ldots, N\right\}$ according to a biased distribution $\operatorname{Pr}\left[c_{r}^{(n)}=c_{s} e^{\frac{j 2 \pi q}{M_{2}}}\right]=\kappa_{q}, q=0,1, \ldots, M_{2}-1$, and replacing (16) by

$$
\boldsymbol{J}_{M, \mathrm{SR}}(\tilde{\boldsymbol{\theta}})_{i, j} \approx \frac{K}{N} \sum_{n=1}^{N}\left(\frac{I_{i}^{(n)} \delta_{i-j}}{\tau_{i}^{2}} \cdot \frac{\tau_{i}}{\kappa_{i}}+\frac{I_{M_{2}-1}^{(n)}}{\tau_{M_{2}-1}^{2}} \cdot \frac{\tau_{M_{2}-1}}{\kappa_{M_{2}-1}}\right),
$$

with $i, j=0,1, \ldots, M_{2}-2$. We select all $\kappa_{q}$ to be much larger than $1 / N$, so that it is unlikely for any $q$ to have $I_{q}^{(n)}=0$ for $n=1,2, \ldots, N$. This yields $\frac{1}{N} \sum_{n=1}^{N} I_{q}^{(n)} \approx \kappa_{q}$ 
for all $q$, so that (17) is close to the analytical result (12). By using IS, we have thus

In the general case, $\boldsymbol{J}(\boldsymbol{\theta})_{i, j}$ from (3) and $\boldsymbol{J}_{M, \mathrm{SRD}}(\overline{\boldsymbol{\theta}})_{i, j}$ from (10) are approximated as

$$
\boldsymbol{J}(\boldsymbol{\theta})_{i, j} \approx \frac{1}{N} \sum_{n=1}^{N} X_{i}\left(\boldsymbol{r}^{(n)} ; \boldsymbol{\theta}\right) X_{j}\left(\boldsymbol{r}^{(n)} ; \boldsymbol{\theta}\right) \cdot \frac{p\left(\boldsymbol{r}^{(n)} ; \boldsymbol{\theta}\right)}{p\left(\boldsymbol{r}^{(n)} ; \zeta\right)}
$$

$$
\boldsymbol{J}_{M, \mathrm{SRD}}(\overline{\boldsymbol{\theta}})_{i, j} \approx \frac{K}{N} \sum_{n=1}^{N} \bar{X}_{i}\left(r_{2}^{(n)}, c_{s} ; \overline{\boldsymbol{\theta}}\right) \bar{X}_{j}\left(r_{2}^{(n)}, c_{s} ; \overline{\boldsymbol{\theta}}\right) \cdot \frac{p\left(r_{2}^{(n)} \mid c_{s} ; \boldsymbol{\theta}\right)}{p\left(r_{2}^{(n)} \mid c_{s} ; \boldsymbol{\eta}\right)},
$$

where $\left\{\boldsymbol{r}^{(n)}, n=1,2, \ldots, N\right\}$ and $\left\{r_{2}^{(n)}, n=1,2, \ldots, N\right\}$ are $\mathrm{N}$ independent realizations generated according to the biased distributions $p\left(\boldsymbol{r}^{(n)} ; \zeta\right)$ and $p\left(r_{2}^{(n)} \mid c_{s} ; \eta\right)$, with

$$
\begin{aligned}
& \zeta=\left(\mathfrak{R}\left(h_{0}\right), \Im\left(h_{0}\right), \kappa_{0}, \kappa_{1}, \ldots, \kappa_{M_{2}-2}, \mathfrak{R}\left(h_{2}\right), \mathfrak{I}\left(h_{2}\right)\right)^{T} \\
& \boldsymbol{\eta}=\left(\kappa_{0}, \kappa_{1}, \ldots, \kappa_{M_{2}-2}, \mathfrak{R}\left(h_{2}\right), \mathfrak{I}\left(h_{2}\right)\right)^{T} .
\end{aligned}
$$

Note that the expression (18) of the FIM is much more time-consuming to evaluate than the expression (19) of the MFIM; indeed, the former requires the generation of observation vectors of $K$ components and the computation of the a posteriori source symbol probabilities, whereas for the latter the observations to be generated are scalar, and no a posteriori source symbol probabilities are needed.

\section{Numerical results}

In this section, the value of the CRBs and MCRBs related to the various channel parameters is obtained for different SNRs. The IS technique from Section 5 has been applied to evaluate the MCRBs. No IS is used when computing the CRBs, due to the complexity involved in evaluating the factor $p\left(\boldsymbol{r}^{(n)} ; \boldsymbol{\theta}\right) / p\left(\boldsymbol{r}^{(n)} ; \boldsymbol{\zeta}\right)$ in (18).

In this section, the value of the CRB and MCRB is obtained for different SNR ratios using the IS technique from Section 5. First, the simulation parameters are specified, whereafter the LBs on the MSEE of the different channel parameters are discussed. The tightness of the obtained LBs is evaluated by comparing the latter with the MSEE of the estimation algorithms from [16], which are briefly discussed in Appendix C.

\subsection{System parameters}

The SD and RD channels are as described in Section 2.1. The actual SR channel is modeled as a flat Rayleigh fading channel with additive white Gaussian noise, characterized by the channel coefficient $h_{1}$. The latter is constant during a frame and has a ZMCSCG distribution with variance $N_{h_{1}}=1 / d_{1}{ }^{{ }^{\text {loss }}}$, with $d_{1}$ the distance between the source and the relay terminal. The noise on the SR channel also has a ZMCSCG distribution with variance $N_{1}$.

The instantaneous SNRs on the SD, SR, and RD channels are $\gamma_{0}=E_{s}\left|h_{0}\right|^{2} / N_{0}, \gamma_{1}=E_{s}\left|h_{1}\right|^{2} / N_{1}$, and $\gamma_{2}=$ $E_{r}\left|h_{2}\right|^{2} / N_{2}$, respectively; the corresponding average SNRs are $E_{s} / N_{0}, E_{s} / N_{1}$ and $E_{r} / N_{2}$. The source, relay, and destination terminals are located at the vertices of an equilateral triangle with normalized edge length, i.e., $d_{0}=d_{1}=$ $d_{2}=1$, yielding $\mathbb{E}\left[\left|h_{n}\right|^{2}\right]=1$ for $n=0,1,2$. All noise variances are taken equal (i.e., $N_{0}=N_{1}=N_{2}$ ). The source symbols and the relay symbols have the same energy (i.e., $\left.E_{s}=E_{r}\right)$.

The source broadcasts BPSK symbols (i.e., $M_{1}=2$ ). The samples received by the relay are quantized to QPSK symbols (i.e., $M_{2}=4$ ) without knowledge of the SR channel, i.e., $c_{r}(k)=e^{j \frac{\pi}{2}\left\lfloor 0.5+\frac{2}{\pi} \arg \left(r_{1}(k)\right)\right\rfloor}$ with $r_{1}(k)$ denoting the $k$ th sample received by the relay. The corresponding transition probabilities of the equivalent SR channel are obtained as function of the SR channel parameters $h_{1}$ and $E_{S} / N_{1}$ using the techniques described in $[10,16]$. For the calculation of the CRB, channel encoding is performed using a $(1,13 / 15)_{8}$ RSCC turbo code [29] that is punctured to a rate of $2 / 3$. A frame contains 1024 information bits, which corresponds to 1536 data symbols after channel encoding and BPSK mapping. In addition to these BPSK data symbols, 12 pilot symbols are added to each frame, to help the destination to obtain estimates for the various channel parameters; this yields a total frame size of $K=1548$ BPSK symbols.

The CRB for a given realization of $\boldsymbol{\theta}$ is obtained by performing the averaging in (4) by means of a MC simulation involving $N=5000$ independent realizations of $r$; this corresponds to the generation of $2 K \cdot N \approx 1.5 \cdot 10^{7}$ scalar observations. Subsequently, $10^{4}$ realizations of $\boldsymbol{\theta}$ are used to average the CRB over $\boldsymbol{\theta}$.

The calculation of the MCRB for a given realization of $\boldsymbol{\theta}$ is performed using MC simulation with IS. The biased transition probabilities $\boldsymbol{\kappa}$ to be used in (19) are generated by assuming that $\gamma_{1}$ is always equal to $0 \mathrm{~dB}$, irrespective of the actual value of $\gamma_{1}$. In doing so, only 5000 realizations of the scalar observation $r_{2}$ that corresponds to $c_{s}=1$ are generated to compute the conditional average over $r_{2}$ in (10). When compared to the $1.5 \cdot 10^{7}$ iterations required for the CRB where no IS is used, IS reduces the simulation time by a factor of 3000 . While the calculation of the (M)CRB is typically not a time-critical application, the simulation time reduction can prove very valuable when prototyping a system, where the changes applied to it can be rapidly benchmarked by means of the MCRB. After averaging over $r_{2}, 10^{4}$ realizations of $\boldsymbol{\theta}$ are used to average the MCRB over $\boldsymbol{\theta}$. 


\subsection{Estimation of the SD channel}

The MSEE of the SD channel coefficient is analyzed as a function of $E_{s} / N_{0}$, while keeping the instantaneous SNR on the SR channel, represented by $\gamma_{1}$, fixed. We set $E_{r} / N_{2}$ equal to $E_{s} / N_{0}$. Figure 2 shows the MSE resulting from the EM algorithm, the CRB and the MCRB related to the estimation of $h_{0}$ for various values of $\gamma_{1}$. The MCRB is independent of $\gamma_{1}$, as was pointed out in Section 4. However, the CRB is affected by the value of $\gamma_{1}$, because $\gamma_{1}$ determines the transition probabilities $\boldsymbol{\tau}$, which in turn impact the a posteriori source symbol probabilities that are used in the computation of the CRB.

As the figure shows, the state of the SR channel, represented by $\gamma_{1}$, has only little impact on the MSEE of the SD channel coefficient. As expected, this effect further diminishes at high SNRs, where the CRB approaches the MCRB.

\subsection{Estimation of the SR channel}

We consider the bounds on the MSEE of the equivalent SR channel transition probabilities $\tau$, as a function of $E_{s} / N_{1}$, with the SD channel being characterized by $E_{s} / N_{0}=E_{s} / N_{1}$. We take fixed values for $\gamma_{2}$, the instantaneous SNR of the RD channel. Figures 3, 4, and 5 show the CRB, the MCRB, and the MSEE resulting from the EM algorithm, for various $\gamma_{2}$. In order to evaluate the impact of an imperfect RD channel estimate $\hat{h_{2}}$ on the estimation performance of the SR channel transition probabilities $\boldsymbol{\tau}$, the aforementioned figures also show the results corresponding to the estimation of $\boldsymbol{\tau}$ when $h_{2}$ is known to the destination. As a LB on the obtained results, the MCRB corresponding to the case of a perfect RD channel (see Section 4.2.1) is also plotted.
Several key observations can be made from the aforementioned figures. At high $\gamma_{2}$ values, shown in Fig. 5, the CRB converges to the MCRB for moderate and high $E_{s} / N_{1}$ when all channels are unknown and, at high $E_{s} / N_{1}$, both the CRB and MCRB converge to the MCRB corresponding to a perfect RD channel. Assuming the $\mathrm{RD}$ channel is known to the destination when estimating, the SR channel yields only a slight decrease in MSE. Figure 5 also shows that at high $\gamma_{2}$ values, the CRB is a tight LB on the MSE of the actual estimation algorithm.

For lower $\gamma_{2}$ (see Figs. 3 and 4), the CRB still approaches the MCRB at high $E_{s} / N_{1}$ when all channels are unknown, but there remains a substantial (especially at very low $\gamma_{2}$ values) gap in MSE as compared to the case where the RD channel is known to the destination; a still larger gap occurs as compared to the case of a perfect RD channel. The former gap is caused by the poor accuracy of $\hat{h_{2}}$ at low $\gamma_{2}$, which in turn deteriorates the estimate of $\boldsymbol{\tau}$, even at large $E_{s} / N_{1}$; this indicates a considerable coupling between the estimates $\hat{h_{2}}$ and $\hat{\boldsymbol{\tau}}$. The difference in MSE between the cases of known $\mathrm{RD}$ channel and perfect RD channel is caused by the noise on the RD channel, and therefore increases with decreasing $\gamma_{2}$.

Next, we observe a MSE floor that occurs at high $E_{s} / N_{1}$ when $\gamma_{2}$ is low. This phenomenon is explained by calculating the MCRB corresponding to the estimation of the SR channel transition probabilities at the high SNR limit. For very high $E_{s} / N_{1}$, the transition probabilities $\tau$ satisfy $\tau_{k} \approx 1$ for a certain index $k=\left\lfloor 0.5+(2 / \pi) \arg \left(h_{1}\right)\right\rfloor$ and $\tau_{l} \approx 0$ for $l \neq k$. Under this assumption, (37) can be written as

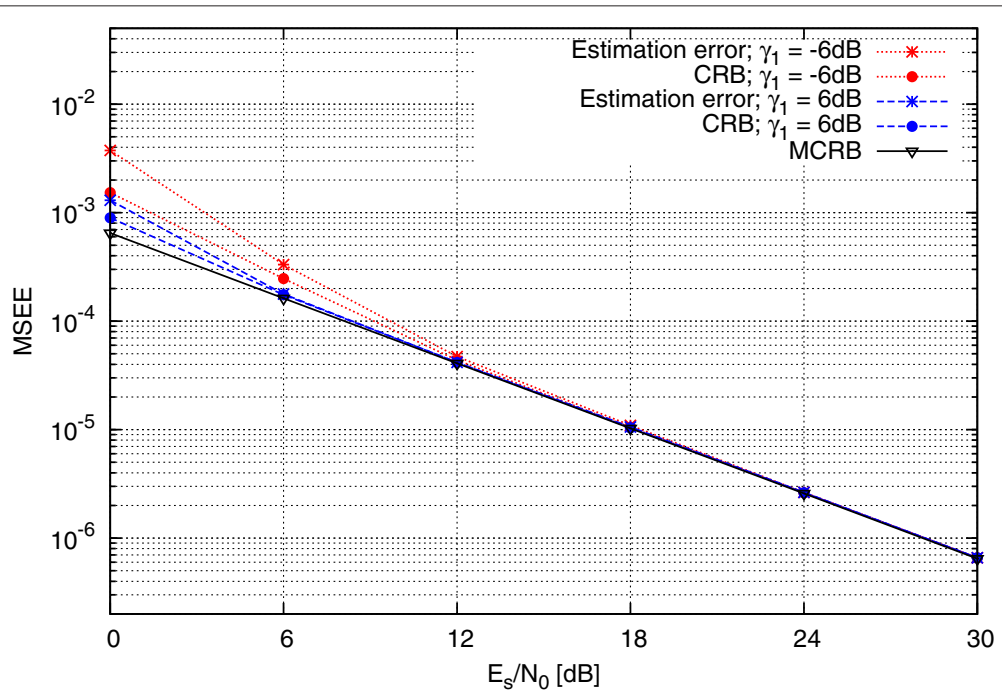

Fig. 2 MSEE of $\hat{h_{0}}$ as a function of the $E_{s} / N_{0}$ ratio for various values of $\gamma_{1}$ 


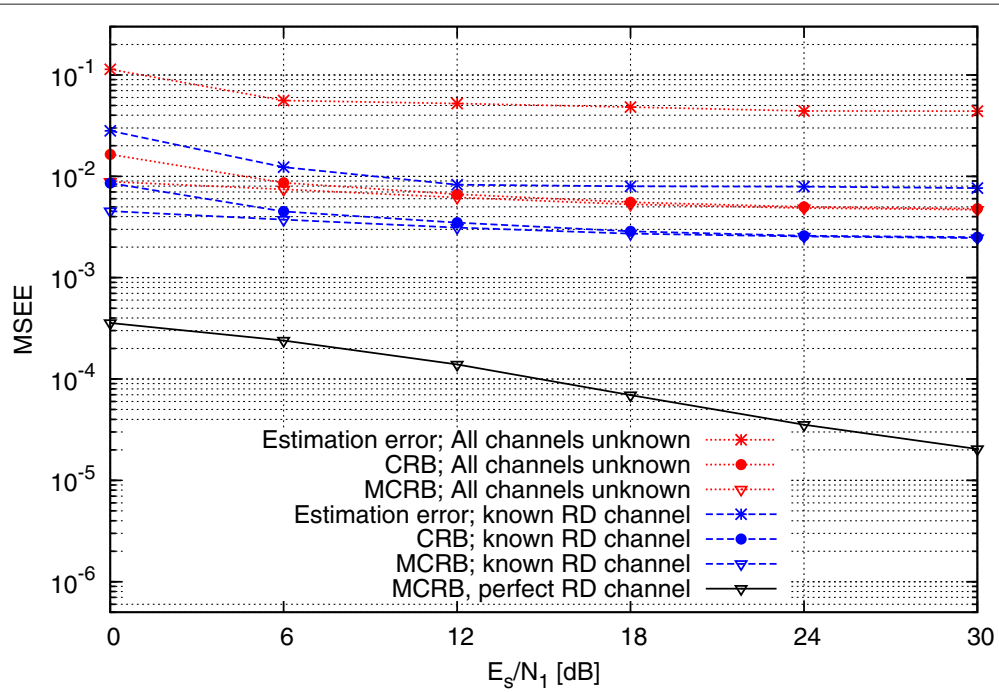

Fig. 3 MSEE of $\hat{\boldsymbol{\tau}}$ as a function of the $E_{s} / N_{1}$ ratio for $\gamma_{2}=-6 \mathrm{~dB}$

$$
\begin{aligned}
& \frac{\partial}{\partial \bar{\theta}_{i}} \ln p\left(r_{2} \mid c_{s} ; \overline{\boldsymbol{\theta}}\right)= \\
& \frac{P\left[r_{2} \mid c_{r}=\chi_{M_{2}}\left(i+\frac{M_{2}}{M_{1}} m\right) ; \overline{\boldsymbol{\theta}}\right]-P\left[r_{2} \mid c_{r}=\chi_{M_{2}}\left(\frac{M_{2}\left(M_{1}+m\right)}{M_{1}}-1\right) ; \overline{\boldsymbol{\theta}}\right]}{P\left[r_{d} \mid c_{r}=\chi_{M_{2}}\left(k+\frac{M_{2}}{M_{1}} m\right) ; \overline{\boldsymbol{\theta}}\right]} .
\end{aligned}
$$

Using (20) when evaluating (9) yields Fig. 6, in which the high $E_{s} / N_{1}$ limit of the MCRB related to estimating $\boldsymbol{\tau}$ is plotted as a function of $\gamma_{2}$. As the figure shows, the value of the MSE floor observed on Figs. 3 and 4 is determined by the instantaneous RD channel quality $\gamma_{2}$. The figure also shows that this MSE floor drops very steeply when $\gamma_{2}>0 \mathrm{~dB}$, explaining the apparent absence of an MSE floor on Fig. 5.

Figures 3, 4 and 5 indicate that the MSEE resulting from the EM algorithm does not approach the CRB at low values of $\gamma_{2}$. This is due to the fact that the estimation scheme described in [16] fails to obtain accurate estimates for the SR transition probabilities at low $\gamma_{2}$, accounting for the gap between the MSEE of the EM algorithm and the (M)CRB.

It should be noted that the inability to accurately estimate the SR transition probabilities at low $\gamma_{2}$ has only a minor effect on the error performance of the decoder at the destination, as observed in [16]. Indeed, at low

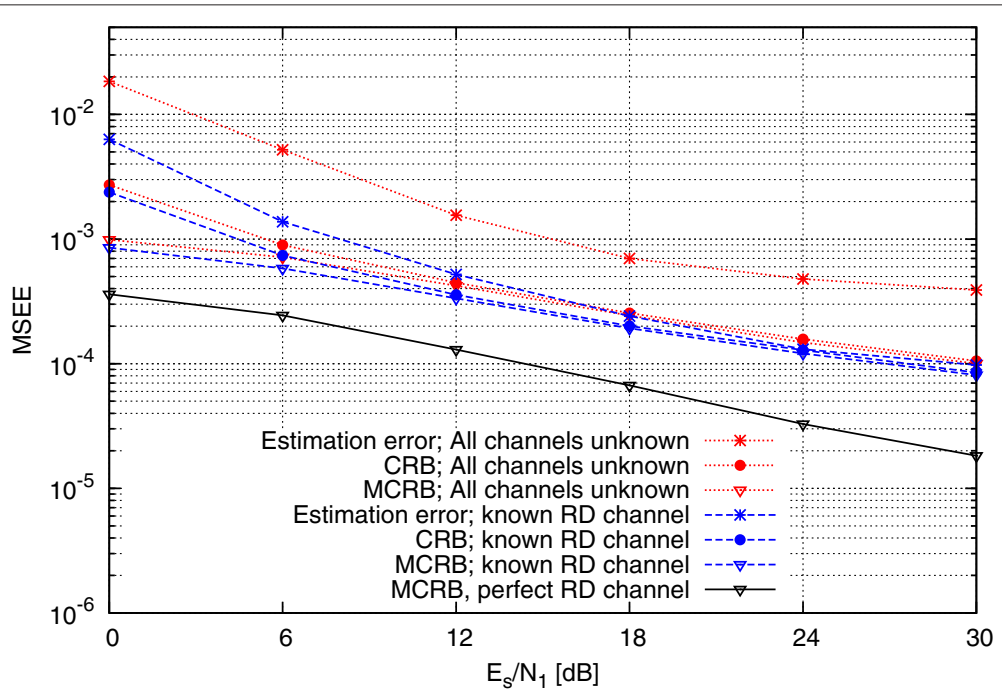

Fig. 4 MSEE of $\hat{\boldsymbol{\tau}}$ as a function of the $E_{s} / N_{1}$ ratio for $\gamma_{2}=0 \mathrm{~dB}$ 


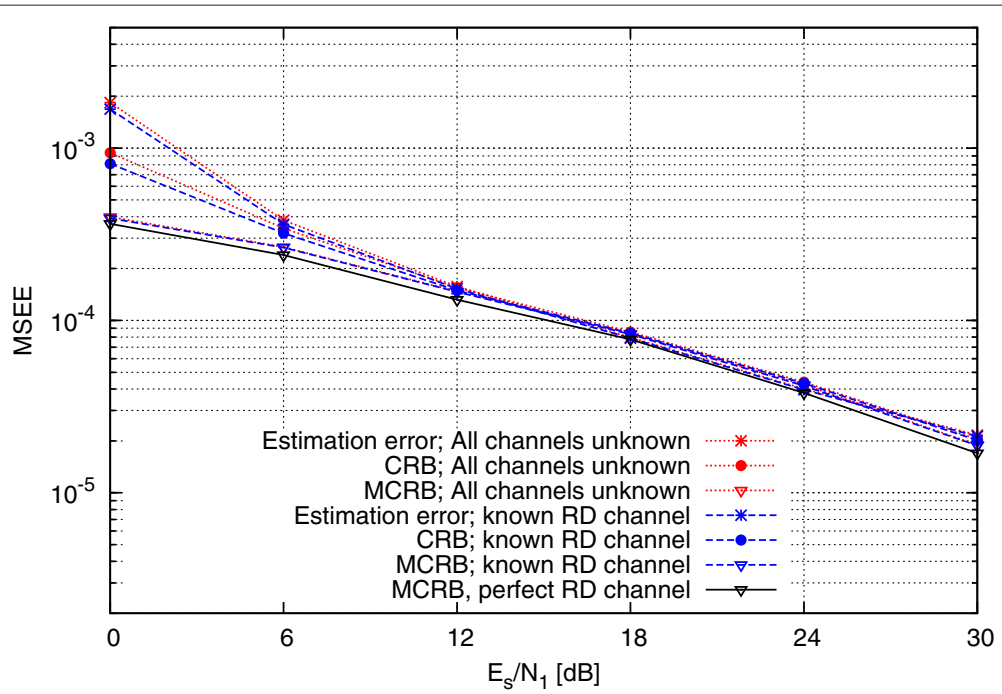

Fig. 5 MSEE of $\hat{\boldsymbol{\tau}}$ as a function of the $E_{s} / N_{1}$ ratio for $\gamma_{2}=6 \mathrm{~dB}$

$\gamma_{2}$, the decoder basically ignores the signal $\boldsymbol{r}_{2}$ received from the relay and exploits only the SD signal $\boldsymbol{r}_{0}$; in this case, the error performance of the decoder is essentially independent of the quality of the SR channel estimate.

\subsection{Estimation of the RD channel}

The MSE resulting from the estimation of the RD channel coefficient is shown in Figs. 7, 8 and 9 as a function of $E_{r} / N_{2}$. We take $E_{s} / N_{0}=E_{r} / N_{2}$, while keeping fixed values for $\gamma_{1}$, the instantaneous SNR on the SR channel. As a reference, the figures also show the results for a known SR channel (i.e., $\hat{\boldsymbol{\tau}}=\boldsymbol{\tau}$ ) and for a perfect SR channel (in which case the relay symbols are deterministically determined by the source symbols).

As the aforementioned figures show, the CRB converges to the MCRB at high $E_{r} / N_{2}$, and this irrespective of $\gamma_{1}$. Unlike the LBs associated with the estimation of the SR channel transition probabilities, the CRB and MCRB of the RD channel coefficient do not exhibit an MSE floor and, for any $\gamma_{1}$, both converge to the (M)CRB corresponding to a known SR channel $(\hat{\boldsymbol{\tau}}=\boldsymbol{\tau})$, and also to the MCRB corresponding to a perfect SR channel. This can be understood by considering $p\left(c_{r}(k) \mid c_{s}(k), r_{2}(k) ; \overline{\boldsymbol{\theta}}\right)$ from (38) for very small $\gamma_{1}$, so that $\tau_{q} \approx \frac{1}{M_{2}}, \forall q$; this yields

$$
p\left(c_{r}(k) \mid r_{2}(k), c_{s}(k) ; \overline{\boldsymbol{\theta}}\right)=\frac{p\left(r_{2}(k) \mid c_{r}(k) ; \overline{\boldsymbol{\theta}}\right)}{\sum_{r_{2}(k)} p\left(r_{2}(k) \mid c_{r}(k) ; \overline{\boldsymbol{\theta}}\right)} .
$$

For large $E_{r} / N_{2}$, we obtain $p\left(c_{r}(k) \mid c_{s}(k), r_{2}(k) ; \overline{\boldsymbol{\theta}}\right) \approx 1$ when $c_{r}(k)$ equals the relay symbol actually transmitted. This indicates that, in spite of the very small $\gamma_{1}$, the relay symbols can be considered known at the destination for large $E_{r} / N_{2}$. This also enables the estimation algorithms discussed in [16] to obtain accurate estimates of $h_{2}$, making the CRB a tight $\mathrm{LB}$ on the estimation performance, especially so at high $E_{r} / N_{2}$ ratios. Hence, seen from an

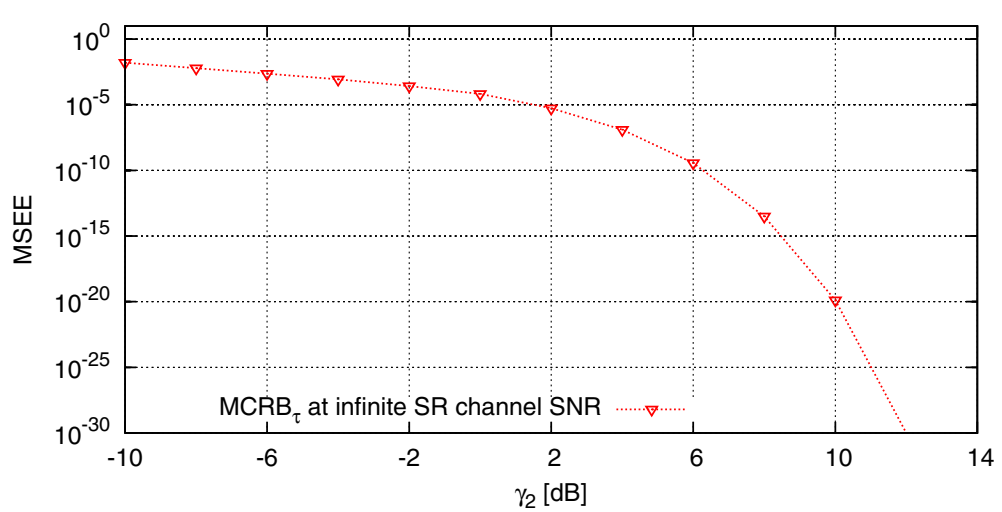

Fig. $6 \mathrm{MCRB}_{\boldsymbol{\tau}}$ at infinite SR channel SNR as function of the instantaneous RD channel SNR 


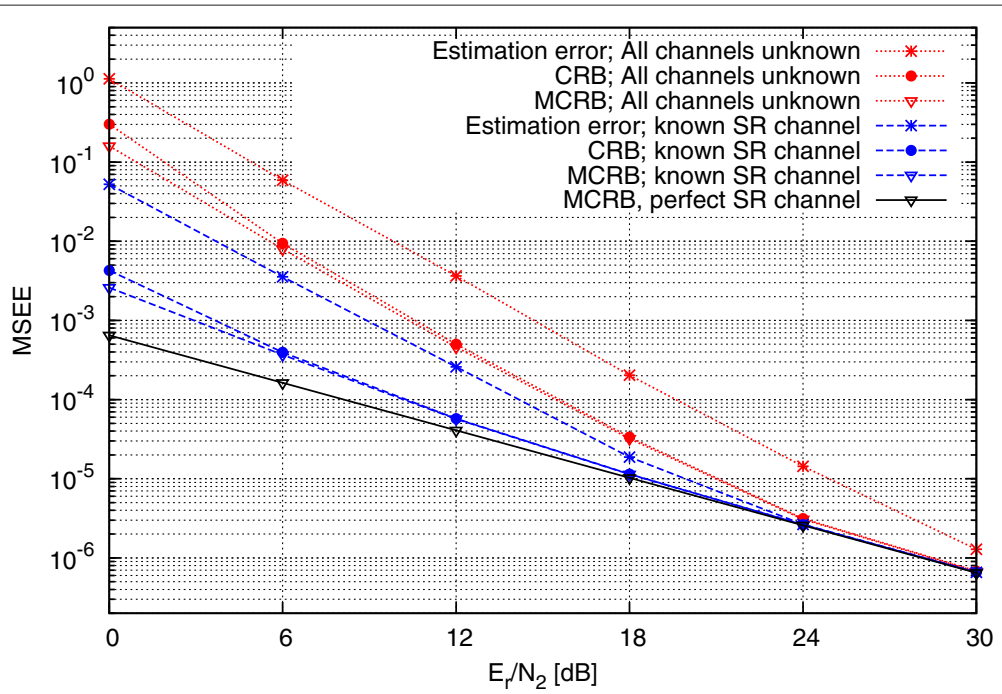

Fig. 7 MSEE of $\hat{h_{2}}$ as a function of the $E_{r} / N_{2}$ ratio for $\gamma_{1}=-6 \mathrm{~dB}$

estimation point of view, it is advantageous to place the relay terminal close to the destination, as this enables the latter to obtain a good estimate of both the SR channel (due to a high average $\gamma_{2}$ value) and the RD channel (due to the robustness of the estimate of $h_{2}$ with respect to low $\gamma_{1}$ values).

Comparison of the results obtained in this subsection to the bounds obtained for the SD channel in Subsection 6.2 indicates that due to the presence of the SR link, the RD channel is more difficult to accurately estimate. This effect diminishes as the SR channel quality is increased and especially if the SR channel is assumed to be known. In the case of a perfect SR channel, the MCRB related to the estimation of the RD channel corresponds to that of the SD channel, as is to be expected.

\section{Conclusions}

In this contribution, the CRB was obtained for all channels in a QF cooperative system. By maintaining a general description of the SR channel and the quantization operation, the obtained results can be applied to a wide variety of cases such as a Rice fading SR channel or a cascade of different channels. In order to reduce the complexity associated with the CRB, we also presented the MCRB, which is a looser bound compared to the CRB but which converges to the latter at high SNR. Except for a few special

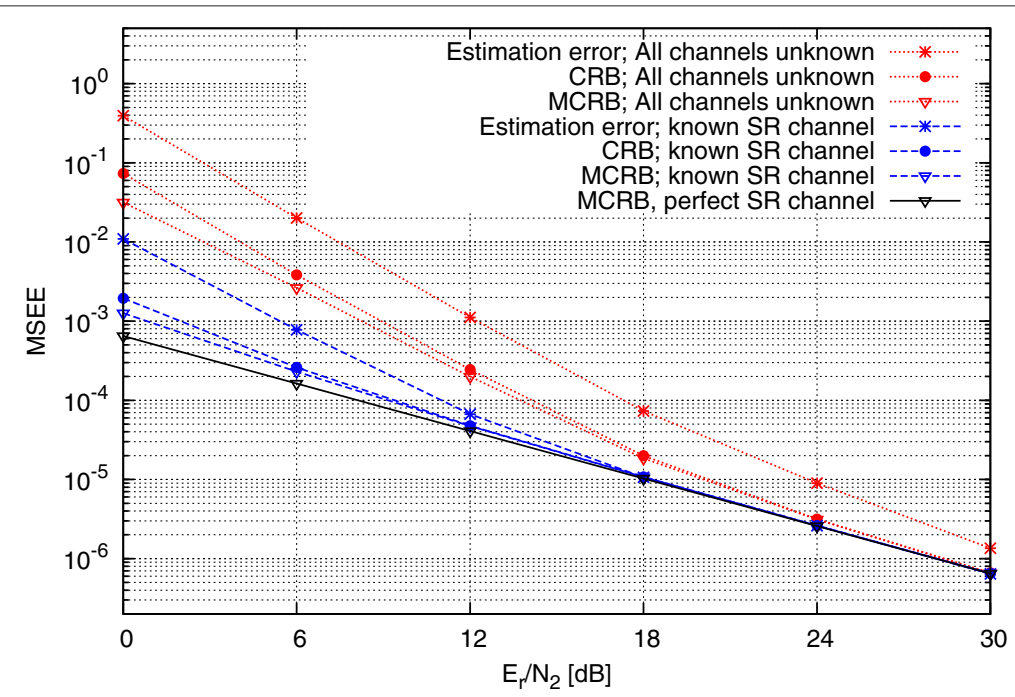

Fig. $8 \mathrm{MSEE}$ of $\hat{h_{2}}$ as a function of the $E_{r} / N_{2}$ ratio for $\gamma_{1}=0 \mathrm{~dB}$ 


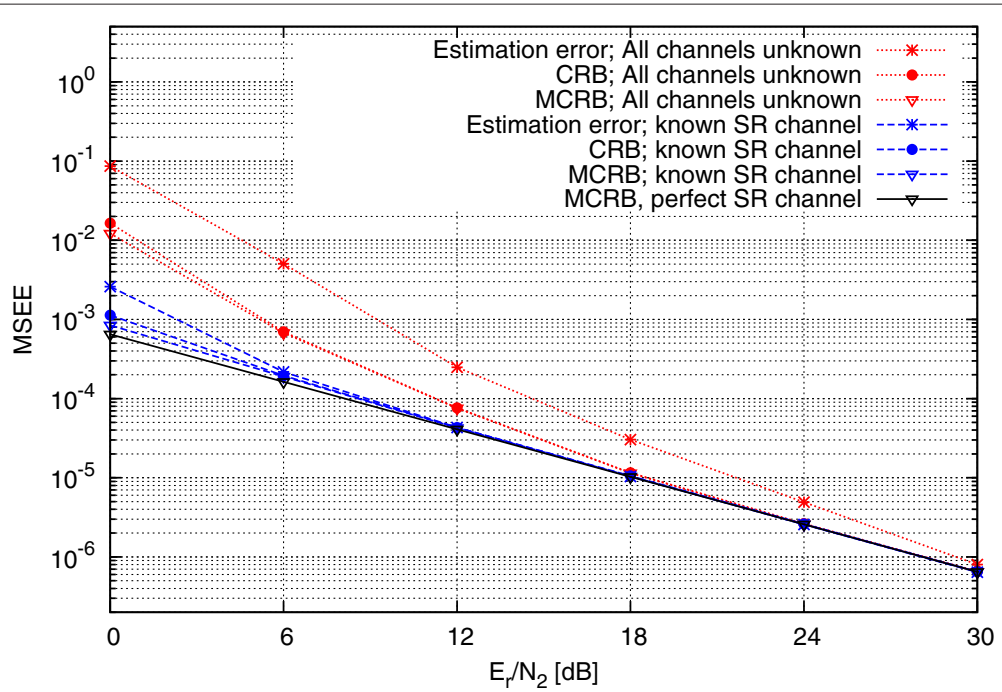

Fig. 9 MSEE of $\hat{h_{2}}$ as a function of the $E_{r} / N_{2}$ ratio for $\gamma_{1}=6 \mathrm{~dB}$

cases in which closed-formed expressions were found for the MCRB, the CRB and MCRB were obtained using numerical simulation methods. By using the proposed IS technique in the numerical computation of the MCRB, the simulation times were substantially reduced, allowing for fast benchmarking when making variations on a system under investigation.

The presented results show that it is possible to obtain accurate estimates of the SD and RD channels, irrespective of the state of the other channels. On the other hand, accurately estimating the SR proves more difficult. The (M)CRB corresponding the estimation of the SR channel exhibits high MSEE values when the RD channel quality is poor. Furthermore, the MSEE of the SR channel parameters is bounded by an error floor, the value of which depends on the state of the RD channel. Fortunately, the decoder performance is virtually unaffected by the accuracy of the SR channel estimate when the RD channel quality is poor.

\section{Notations}

a Scalar

a Column vector

A Matrix

$\mathbf{0}_{N}$ Row vector of length $N$ with all elements equal to 0

$\mathbf{1}_{N}$ Row vector of length $N$ with all elements equal to 1

$\mathrm{I}_{N}$ Identity matrix of rank $N$

(.) ${ }^{T},(.)^{H}$ Transpose and Hermitian transpose

$\operatorname{Tr}$ (.) Matrix trace

(.) $)^{*}$ Complex conjugate

|.| Absolute value

$\mathfrak{R}(),. \mathfrak{I}($.$) Real and imaginary part$

$\mathbb{E}_{\mathbf{x}}[$.] Expectation with respect to the random vector $\boldsymbol{x}$

\section{Appendix A: Calculation of the FIM}

In the following section, the calculation of the elements of the FIM is outlined. These elements are obtained by evaluating

$$
\begin{aligned}
J(\boldsymbol{\theta})_{i, j} & =\mathbb{E}_{\boldsymbol{r}}\left[\frac{\partial}{\partial \theta_{i}} \ln p(\boldsymbol{r} ; \boldsymbol{\theta}) \frac{\partial}{\partial \theta_{j}} \ln p\left(\boldsymbol{r}_{2} ; \boldsymbol{\theta}\right)\right] \\
& =\mathbb{E}_{\boldsymbol{r}}\left[X_{i}(\boldsymbol{r} ; \boldsymbol{\theta}) X_{j}(\boldsymbol{r} ; \boldsymbol{\theta})\right]
\end{aligned}
$$

with $X_{i}(\boldsymbol{r} ; \boldsymbol{\theta})=\frac{\partial}{\partial \theta_{i}} \ln p(\boldsymbol{r} ; \boldsymbol{\theta})$. By conditioning on the symbols $c_{s}$ and $\boldsymbol{c}_{r}$ sent by the source and the relay, $X_{i}$ can be written as

$$
\begin{aligned}
X_{i}(\boldsymbol{r} ; \boldsymbol{\theta}) & =\frac{\partial}{\partial \theta_{i}} \ln \sum_{\boldsymbol{c}_{s}, \boldsymbol{c}_{r}} p\left(\boldsymbol{r} \mid \boldsymbol{c}_{r}, \boldsymbol{c}_{s} ; \boldsymbol{\theta}\right) p\left(\boldsymbol{c}_{r} \mid \boldsymbol{c}_{s} ; \boldsymbol{\theta}\right) p\left(\boldsymbol{c}_{s}\right) \\
& =\sum_{\boldsymbol{c}_{s}, \boldsymbol{c}_{r}} F\left(\boldsymbol{r}, \boldsymbol{c}_{r}, \boldsymbol{c}_{s} ; \boldsymbol{\theta}\right) p\left(\boldsymbol{c}_{r}, \boldsymbol{c}_{s} \mid \boldsymbol{r} ; \boldsymbol{\theta}\right) \\
& =\mathbb{E}_{\boldsymbol{c}_{s}, \boldsymbol{c}_{r} \mid \boldsymbol{r}}\left[F\left(\boldsymbol{r}, \boldsymbol{c}_{r}, \boldsymbol{c}_{s} ; \boldsymbol{\theta}\right)\right]
\end{aligned}
$$

where

$$
F\left(\boldsymbol{r}, \boldsymbol{c}_{r}, \boldsymbol{c}_{s} ; \boldsymbol{\theta}\right)=\frac{\partial}{\partial \theta_{i}} \ln p\left(\boldsymbol{r} \mid \boldsymbol{c}_{r}, \boldsymbol{c}_{s} ; \boldsymbol{\theta}\right)+\frac{\partial}{\partial \theta_{i}} \ln p\left(\boldsymbol{c}_{r} \mid \boldsymbol{c}_{s} ; \boldsymbol{\theta}\right) .
$$

Taking into account that

$$
p\left(\boldsymbol{r} \mid \boldsymbol{c}_{r}, \boldsymbol{c}_{s} ; \boldsymbol{\theta}\right)=p\left(\boldsymbol{r}_{0} \mid \boldsymbol{c}_{s} ; h_{0}\right) p\left(\boldsymbol{r}_{2} \mid \boldsymbol{c}_{r} ; h_{2}\right)
$$

and $p\left(\boldsymbol{c}_{r} \mid \boldsymbol{c}_{S} ; \boldsymbol{\theta}\right)$ depends only on the transition probabilities of the equivalent SR channel, we obtain 


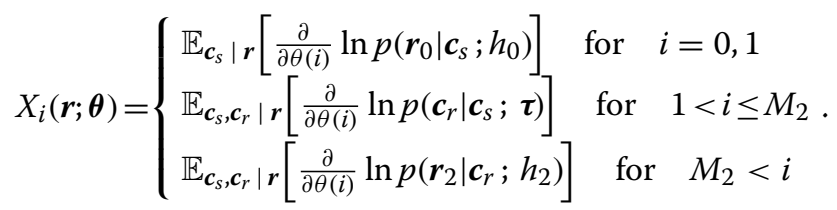

These expressions can be expanded as

$$
\begin{aligned}
& X_{i}(\boldsymbol{r} ; \boldsymbol{\theta})= \\
& \left\{\begin{array}{r}
\sum_{k=1}^{K} \sum_{c_{s}(k)} \frac{\partial}{\partial \theta(i)} \ln p\left(r_{0}(k) \mid c_{s}(k) ; h_{0}\right) p\left(c_{s}(k) \mid \boldsymbol{r} ; \boldsymbol{\theta}\right) \\
\text { for } i=0,1 \\
\sum_{k=1}^{K} \sum_{c_{s}(k), c_{r}(k)} \frac{\partial}{\partial \theta(i)} \ln p\left(c_{r}(k) \mid c_{s}(k) ; \boldsymbol{\tau}\right) p\left(c_{s}(k), c_{r}(k) \mid \boldsymbol{r} ; \boldsymbol{\theta}\right) \\
\text { for } 1<i \leq M_{2} \\
\sum_{k=1}^{K} \sum_{c_{s}(k), c_{r}(k)} \frac{\partial}{\partial \theta(i)} \ln p\left(r_{2}(k) \mid c_{r}(k) ; h_{2}\right) p\left(c_{s}(k), c_{r}(k) \mid \boldsymbol{r} ; \boldsymbol{\theta}\right) \\
\text { for } M_{2}<i
\end{array}\right.
\end{aligned}
$$

where the summations over $c_{s}(k)$ and $c_{r}(k)$ run over all points in their respective constellations. In (25), the a posteriori probabilities $p\left(c_{s}(k) \mid \boldsymbol{r} ; \boldsymbol{\theta}\right)$ of the source symbols depend on the structure of the channel code, and are obtained from a soft decoder that operates on $\boldsymbol{r}$. The joint a posteriori probabilities $p\left(c_{s}(k), c_{r}(k) \mid \boldsymbol{r} ; \boldsymbol{\theta}\right)$ in (25) can be decomposed as

$p\left(c_{s}(k), c_{r}(k) \mid \boldsymbol{r} ; \boldsymbol{\theta}\right)=p\left(c_{r}(k) \mid c_{s}(k), r_{2}(k) ; \boldsymbol{\theta}\right) p\left(c_{s}(k) \mid \boldsymbol{r} ; \boldsymbol{\theta}\right)$.

The probabilities $p\left(c_{r}(k) \mid c_{s}(k), r_{2}(k) ; \boldsymbol{\theta}\right)$ are obtained as

$$
\begin{aligned}
& p\left(c_{r}(k) \mid c_{s}(k), r_{2}(k) ; \boldsymbol{\theta}\right) \\
& \quad=\frac{p\left(r_{2}(k) \mid c_{r}(k) ; h_{2}\right) p\left(c_{r}(k) \mid c_{s}(k) ; \boldsymbol{\tau}\right)}{\sum_{\alpha} p\left(r_{2}(k) \mid c_{r}(k)=\alpha ; h_{2}\right) P\left[c_{r}(k)=\alpha \mid c_{s}(k) ; \boldsymbol{\tau}\right]} .
\end{aligned}
$$

The derivative in the first line of (25) reduces to

$\frac{\partial}{\partial \theta_{i}} \ln p\left(r_{0}(k) \mid c_{s}(k) ; h_{0}\right)= \begin{cases}\frac{2}{N_{0}} \sqrt{E_{s}} \Re\left(r_{0}(k) c_{s}(k)^{*}-\sqrt{E_{s}} h_{0}\right) & i=0 \\ \frac{2}{N_{0}} \sqrt{E_{s}} \Im\left(r_{0}(k) c_{s}(k)^{*}-\sqrt{E_{s}} h_{0}\right) & i=1\end{cases}$

and a similar expression holds for the derivative in the third line of (25). The derivative in the second line of (25) is expressed as

$$
\begin{aligned}
& \frac{\partial}{\partial \theta(i)} \ln p\left(c_{r}(k) \mid c_{s}(k) ; \boldsymbol{\tau}\right) \\
= & \frac{\partial}{\partial \theta(i)} \ln P\left[c_{r}(k)=\chi_{M_{2}}\left(q+\frac{M_{2}}{M_{1}} m\right) \mid c_{s}(k)=\chi_{M_{1}}(m) ; \boldsymbol{\tau}\right] \\
= & \left\{\begin{array}{lrr}
\frac{1}{\tau_{q}} & \text { for } & q=i-2 \\
\frac{-1}{\tau_{M_{2}-1}} & \text { for } & q=M_{2}-1 . \\
0 & & \text { otherwise }
\end{array}\right.
\end{aligned}
$$

Based on the above expressions, the quantities $X_{i}(\boldsymbol{r} ; \boldsymbol{\theta})$ can be evaluated for any $(r, \theta)$; the associated computational complexity is rather high, because the calculation of the a posteriori source symbol probabilities $p\left(c_{s}(k) \mid \boldsymbol{r} ; \boldsymbol{\theta}\right)$ requires a soft decoding operation.

\section{Appendix B: Calculation of the MFIM}

The elements of the MFIM are defined by (9) and are equal to

$$
\begin{aligned}
\boldsymbol{J}_{M}(\boldsymbol{\theta})_{i, j} & =\mathbb{E}_{\boldsymbol{r} \mid \boldsymbol{c}_{s}}\left[\frac{\partial}{\partial \theta_{i}} \ln p\left(\boldsymbol{r} \mid \boldsymbol{c}_{s} ; \boldsymbol{\theta}\right) \frac{\partial}{\partial \theta_{j}} \ln p\left(\boldsymbol{r} \mid \boldsymbol{c}_{s} ; \boldsymbol{\theta}\right)\right] \\
& =\mathbb{E}_{\boldsymbol{r} \mid \boldsymbol{c}_{s}}\left[X_{i}\left(\boldsymbol{r}, \boldsymbol{c}_{s} ; \boldsymbol{\theta}\right) X_{j}\left(\boldsymbol{r}, \boldsymbol{c}_{s} ; \boldsymbol{\theta}\right)\right],
\end{aligned}
$$

with $X_{i}\left(\boldsymbol{r}, \boldsymbol{c}_{s} ; \boldsymbol{\theta}\right)=\frac{\partial}{\partial \theta_{i}} \ln p\left(\boldsymbol{r} \mid \boldsymbol{c}_{s} ; \boldsymbol{\theta}\right)$. As $p\left(\boldsymbol{r} \mid \boldsymbol{c}_{r}, \boldsymbol{c}_{s} ; \boldsymbol{\theta}\right)=$ $p\left(\boldsymbol{r}_{0} \mid \boldsymbol{c}_{s} ; \boldsymbol{\theta}\right) p\left(\boldsymbol{r}_{2} \mid \boldsymbol{c}_{r} ; \boldsymbol{\theta}\right), X_{i}$ can be written as

$$
X_{i}\left(\boldsymbol{r}, \boldsymbol{c}_{s} ; \boldsymbol{\theta}\right)=\frac{\partial}{\partial \theta_{i}} \ln p\left(\boldsymbol{r}_{0} \mid \boldsymbol{c}_{s} ; \boldsymbol{\theta}\right)+\frac{\partial}{\partial \theta_{i}} \ln p\left(\boldsymbol{r}_{2} \mid \boldsymbol{c}_{s} ; \boldsymbol{\theta}\right) .
$$

As $p\left(\boldsymbol{r}_{0} \mid \boldsymbol{c}_{s} ; \boldsymbol{\theta}\right)$ and $p\left(\boldsymbol{r}_{2} \mid \boldsymbol{c}_{r}, \boldsymbol{c}_{s} ; \boldsymbol{\theta}\right)$ depend only on $h_{0}$ and $\left(\boldsymbol{\tau}, h_{2}\right)$, respectively, the first term in (29) is zero for $i>1$, and the second term is zero for $i \leq 1$. Substituting (29) in (28) and evaluating the terms for which $\theta_{i}$ is a parameter of the SD channel and $\theta_{j}$ is a parameter of the SRD channel (i.e., $i \leq 1, j>1$ ) yields

$$
\begin{aligned}
\boldsymbol{J}_{M}(\boldsymbol{\theta})_{i, j} & =\mathbb{E}_{\boldsymbol{r} \mid \boldsymbol{c}_{s}}\left[\frac{\partial}{\partial \theta_{i}} \ln p\left(\boldsymbol{r}_{0} \mid \boldsymbol{c}_{\boldsymbol{s}} ; \boldsymbol{\theta}\right) \frac{\partial}{\partial \theta_{j}} \ln p\left(\boldsymbol{r}_{2} \mid \boldsymbol{c}_{s} ; \boldsymbol{\theta}\right)\right] \\
& =\iint \frac{\partial}{\partial \theta_{i}} \ln p\left(\boldsymbol{r}_{0} \mid \boldsymbol{c}_{s} ; \boldsymbol{\theta}\right) \frac{\partial}{\partial \theta_{j}} \ln p\left(\boldsymbol{r}_{2} \mid \boldsymbol{c}_{s} ; \boldsymbol{\theta}\right) p\left(\boldsymbol{r} \mid \boldsymbol{c}_{s} ; \boldsymbol{\theta}\right) \mathrm{d} \boldsymbol{r}_{0} \mathrm{~d} \boldsymbol{r}_{2} \\
& =\int \frac{\partial}{\partial \theta_{i}} p\left(\boldsymbol{r}_{0} \mid \boldsymbol{c}_{s} ; \boldsymbol{\theta}\right) \mathrm{d} \boldsymbol{r}_{0} \int \frac{\partial}{\partial \theta_{j}} p\left(\boldsymbol{r}_{2} \mid \boldsymbol{c}_{s} ; \boldsymbol{\theta}\right) \mathrm{d} \boldsymbol{r}_{2}=0,
\end{aligned}
$$

so that $\boldsymbol{J}_{M}(\boldsymbol{\theta})$ is a block-diagonal matrix which we represent as

$$
\boldsymbol{J}_{M}=\left[\begin{array}{cc}
\boldsymbol{J}_{M, \mathrm{SD}} & 0 \\
0 & \boldsymbol{J}_{M, \mathrm{SRD}}
\end{array}\right] .
$$

It can be shown from (27) that $\boldsymbol{J}_{M, \mathrm{SD}}=\left(\frac{2 K E_{s}}{N_{0}}\right) \mathbf{I}_{2}$. By grouping the parameters from the SRD channel into the vector $\overrightarrow{\boldsymbol{\theta}}=\left\{\theta_{i}, i>1\right\}$ we obtain

$$
\frac{\partial}{\partial \bar{\theta}} \ln p\left(\boldsymbol{r}_{2} \mid \boldsymbol{c}_{s} ; \overline{\boldsymbol{\theta}}\right)=\sum_{k=1}^{K} \frac{\partial}{\partial \bar{\theta}_{i}} \ln p\left(r_{2}(k) \mid c_{s}(k) ; \overline{\boldsymbol{\theta}}\right),
$$

with

$p\left(r_{2}(k) \mid c_{s}(k) ; \overline{\boldsymbol{\theta}}\right)=\sum_{c_{r}(k)} p\left(r_{2}(k) \mid c_{r}(k) ; \overline{\boldsymbol{\theta}}\right) p\left(c_{r}(k) \mid c_{s}(k) ; \overline{\boldsymbol{\theta}}\right)$,

where the summation over $c_{r}(k)$ runs over points of the $M_{2}$-PSK constellation. Using (31), the elements of $\boldsymbol{J}_{M, \mathrm{SRD}}$ can be written as 
$\boldsymbol{J}_{M, \mathrm{SRD}}(\overline{\boldsymbol{\theta}})_{i, j}=$

$\mathbb{E}_{\boldsymbol{r}_{2} \mid c_{s}}\left[\sum_{k, \tilde{k}=1}^{K} \frac{\partial}{\partial \bar{\theta}_{i}} \ln p\left(r_{2}(k) \mid c_{s}(k) ; \overline{\boldsymbol{\theta}}\right) \frac{\partial}{\partial \bar{\theta}_{j}} \ln p\left(r_{2}(\tilde{k}) \mid c_{s}(\tilde{k}) ; \overline{\boldsymbol{\theta}}\right)\right]$.

Evaluation of the terms for which $k \neq \tilde{k}$ yields

$$
\begin{aligned}
& \mathbb{E}_{\boldsymbol{r}_{2} \mid \boldsymbol{c}_{s}}\left[\frac{\partial}{\partial \bar{\theta}_{i}} \ln p\left(r_{2}(k) \mid c_{s}(k) ; \overline{\boldsymbol{\theta}}\right) \frac{\partial}{\partial \bar{\theta}_{j}} \ln p\left(r_{2}(\tilde{k}) \mid c_{s}(\tilde{k}) ; \overline{\boldsymbol{\theta}}\right)\right]= \\
& \mathbb{E}_{r_{2}(k) \mid c_{s}(k)}\left[\frac{\partial}{\partial \bar{\theta}_{i}} \ln p\left(r_{2}(k) \mid c_{s}(k) ; \overline{\boldsymbol{\theta}}\right)\right] \mathbb{E}_{r_{2}(\tilde{k}) \mid c_{s}(\tilde{k})}\left[\frac{\partial}{\partial \bar{\theta}_{j}} \ln p\left(r_{2}(\tilde{k}) \mid c_{s}(\tilde{k}) ; \overline{\boldsymbol{\theta}}\right)\right],
\end{aligned}
$$

with

$\mathbb{E}_{r_{2}(k) \mid c_{s}(k)}\left[\frac{\partial}{\partial \bar{\theta}_{i}} \ln p\left(r_{2}(k) \mid c_{s}(k) ; \overline{\boldsymbol{\theta}}\right)\right]=\int \frac{\partial}{\partial \bar{\theta}_{i}} p\left(r_{2}(k) \mid c_{s}(k) ; \overline{\boldsymbol{\theta}}\right) \mathrm{d} r_{2}(k)=0$.

Substituting (33), (34) into (32) yields

$$
\begin{aligned}
& \boldsymbol{J}_{M, S R D}(\overline{\boldsymbol{\theta}})_{i, j}= \\
& \sum_{k=1}^{K} \mathbb{E}_{r_{2}(k) \mid c_{s}(k)}\left[\frac{\partial}{\partial \bar{\theta}_{i}} \ln p\left(r_{2}(k) \mid c_{s}(k) ; \overline{\boldsymbol{\theta}}\right) \frac{\partial}{\partial \bar{\theta}_{j}} \ln p\left(r_{2}(k) \mid c_{s}(k) ; \overline{\boldsymbol{\theta}}\right)\right] \\
& =K \mathbb{E}_{r_{2}(0) \mid c_{s}(0)}\left[\bar{X}_{i}\left(r_{2}, c_{s} ; \overline{\boldsymbol{\theta}}\right) \bar{X}_{j}\left(r_{2}, c_{s} ; \overline{\boldsymbol{\theta}}\right)\right],
\end{aligned}
$$

where

$$
\bar{X}_{i}\left(r_{2}, c_{s} ; \overline{\boldsymbol{\theta}}\right)=\frac{\partial}{\partial \bar{\theta}_{i}} \ln p\left(r_{2} \mid c_{s} ; \overline{\boldsymbol{\theta}}\right)
$$

and $r_{2}$ and $c_{s}$ are short-hand notations for $r_{2}(0)$ and $c_{s}(0)$. The functions $\bar{X}_{i}\left(r_{2}, c_{s} ; \overline{\boldsymbol{\theta}}\right)$ can further be expressed as

$$
\begin{aligned}
& \bar{X}_{i}\left(r_{2}, c_{s} ; \overline{\boldsymbol{\theta}}\right)= \\
& \frac{p\left[r_{2} \mid c_{r}=\chi_{M_{2}}\left(i+\frac{M_{2}}{M_{1}} m\right) ; \overline{\boldsymbol{\theta}}\right]-p\left[r_{2} \mid c_{r}=\chi_{M_{2}}\left(\frac{M_{2}\left(M_{1}+m\right)}{M_{1}}-1\right) ; \overline{\boldsymbol{\theta}}\right]}{p\left(r_{2} \mid c_{s}=\chi_{M_{1}}(m)\right)}
\end{aligned}
$$

for $0<i \leq M_{2}-2$ and

$$
\bar{X}_{i}\left(r_{2}, c_{s} ; \overline{\boldsymbol{\theta}}\right)=\sum_{c_{r}} \frac{\partial}{\partial \bar{\theta}_{i}} \ln p\left(r_{2} \mid c_{r} ; \overline{\boldsymbol{\theta}}\right) p\left(c_{r} \mid r_{2}, c_{s} ; \overline{\boldsymbol{\theta}}\right)
$$

for $i>M_{2}-2$. Having obtained closed-form expressions for $\bar{X}_{i}\left(r_{2}, c_{s} ; \overline{\boldsymbol{\theta}}\right)$, the expectation in (35) is evaluated by means of the importance sampling method outlined in Section 5 .

\section{Appendix C: Estimation of the unknown channel parameters}

In order to evaluate the tightness of the obtained lower bounds, the latter are compared to the MSE results from the estimation algorithms described in [16], where maximum likelihood (ML) pilot-based estimates of the various channel parameters are refined using the expectationmaximization (EM) algorithm. In this section, we will briefly outline the estimation process. The reader is referred to [16] for further reading. When using the channel model outlined in Section 6.1, the likelihood of the symbols received at the destination is equal to

$$
\begin{aligned}
& p\left(\boldsymbol{r}_{0}, \boldsymbol{r}_{2} \mid \boldsymbol{c}_{s}, h_{0}, h_{2}, \boldsymbol{\tau}\right) \\
& \quad=\prod_{k=1}^{K} p\left(r_{0}(k) \mid c_{s}(k), h_{0}\right) \sum_{c_{r}(k)} p\left(r_{2}(k) \mid c_{r}(k), h_{2}\right) p\left(c_{r}(k) \mid c_{s}(k), \boldsymbol{\tau}\right),
\end{aligned}
$$

with

$$
\begin{aligned}
& p\left(r_{0}(k) \mid c_{s}(k), h_{0}, N_{0}\right)=\frac{1}{\pi N_{0}} \exp \left(-\frac{\left|r_{0}(k)-h_{0} c_{s}(k)\right|^{2}}{N_{0}}\right) \\
& p\left(r_{2}(k) \mid c_{r}(k), h_{2}, N_{2}\right)=\frac{1}{\pi N_{2}} \exp \left(-\frac{\left|r_{2}(k)-h_{2} c_{r}(k)\right|^{2}}{N_{2}}\right) .
\end{aligned}
$$

The ML estimates of the parameters $h_{0}, h_{2}$ and $\tau$ are calculated in two steps. In a first step, pilot-based estimates of these parameters are obtained. To this purpose, $K_{p}$ pilot symbols are added to data symbols broadcast by the source in the first timeslot, resulting in frames consisting of $K+K_{p}$ symbols. These pilot symbols are received by the destination, where they are used to calculate an estimate of $h_{0}$. They are also received by the relay, where they are quantized together with the data symbols, and are broadcast to the destination in the second timeslot. The destination uses the pilot symbols received from relay to calculate an estimate of $\boldsymbol{\tau}$ and $h_{2}$. Using $\boldsymbol{c}_{s p}$ to denote the pilot symbols transmitted by the source, and $\boldsymbol{r}_{0 p}$ and $\boldsymbol{r}_{2 p}$ to denote the part of $\boldsymbol{r}_{0}$ and $\boldsymbol{r}_{2}$, respectively, that corresponds to the received pilot symbols, the pilot-based ML estimates of the unknown channel parameters are obtained by solving the following equations:

$$
\begin{gathered}
\hat{h}_{0, p}=\arg \max _{h_{0}} p\left(\boldsymbol{r}_{0 p} \mid h_{0}, \boldsymbol{c}_{s p}\right) \\
\left(\hat{\boldsymbol{\tau}_{p}}, \hat{h}_{2 p}\right)=\arg \max _{\left(\boldsymbol{\tau}, h_{2}\right)} p\left(\boldsymbol{r}_{2 p} \mid \boldsymbol{\tau}, h_{2}, \boldsymbol{c}_{s p}\right) .
\end{gathered}
$$

The first equation from (39) can be solved analytically, while the second is solved using the EM algorithm, where the quantized pilot symbols transmitted by the relay are considered to be nuisance parameters. The reader is referred to [16], section III-C, for a detailed resolution of (39).

After pilot-based estimates of $h_{0}, h_{2}$ and $\boldsymbol{\tau}$ are obtained, they are refined using a code-aided EM approach. To this purpose, the data symbols transmitted by the source and the quantized symbols transmitted by the relay are considered to be nuisance parameters. Each EM iteration $i$, refined estimates of $h_{0}, h_{2}$ and $\tau$ are obtained by solving the following equation: 


$$
\left({\hat{h_{0}}}^{(i)},{\hat{h_{2}}}^{(i)}, \hat{\boldsymbol{\tau}}^{(i)}\right)=\arg \max _{\left(h_{0}, h_{2}, \tau\right)} Q\left(h_{0}, h_{2}, \boldsymbol{\tau},{\hat{h_{0}}}^{(i-1)},{\hat{h_{2}}}^{(i-1)}, \hat{\boldsymbol{\tau}}^{(i-1)}\right)
$$

with

$$
\begin{aligned}
& Q\left(h_{0}, h_{2}, \boldsymbol{\tau},{\hat{h_{0}}}^{(i-1)},{\hat{h_{2}}}^{(i-1)}, \hat{\boldsymbol{\tau}}^{(i-1)}\right)=\mathrm{E}_{\boldsymbol{c}_{s}, \boldsymbol{c}_{r}} \\
& {\left[\ln p\left(\boldsymbol{r}_{0}, \boldsymbol{r}_{2} \mid \boldsymbol{c}_{s}, \boldsymbol{c}_{r}, h_{0}, h_{2}\right)+\ln p\left(\boldsymbol{c}_{r} \mid \boldsymbol{c}_{s}, \boldsymbol{\tau}\right) \mid \boldsymbol{r}_{0}, \boldsymbol{r}_{2},{\hat{h_{0}}}^{(i-1)},{\hat{h_{2}}}^{(i-1)}, \hat{\boldsymbol{\tau}}^{(i-1)}\right] .}
\end{aligned}
$$

The reader is referred to [16], section III-B, for the solution of (40). Using the results from [16], it can easily be shown that the obtained estimates of $h_{0}, h_{2}$ and $\tau$ are unbiased, so that the CRB is indeed a LB for the considered estimates.

\section{Competing interests}

The authors declare that they have no competing interests.

\section{Acknowledgements}

The authors wish to acknowledge the Agency for Innovation by Science and Technology Flanders (IWT Belgium) that motivated this work. This research has been funded by the Interuniversity Attraction Poles Programme initiated by the Belgian Science Policy Office.

\section{Received: 20 April 2015 Accepted: 6 October 2015}

Published online: 19 October 2015

\section{References}

1. B Sklar, Rayleigh fading channels in mobile digital communication systems part l: characterization. IEEE Commun. Mag. 35(7), 90-100 (1997)

2. TM Cover, JA Thomas, Elements of Information Theory. (Wiley, New York, 1991)

3. L Cimini Jr, Analysis and simulation of a digital mobile channel using orthogonal frequency-division multiplexing. EEE Trans. on Commun. 33(7), 665-675 (1985)

4. GJ Foschini, MJ Gans, On limits of wireless communications in a fading environment when using multiple antennas. Wirel. Pers. Commun. 6(3), 311-335 (1998)

5. G Foschini, Layered space time architecture for wireless communication in a fading environment when using multi-element antennas. Bell Labs. Tech. 1(2), 41-59 (1996)

6. A Sendonaris, E Erkip, B Aazhang, User cooperation diversity - part l:system Description. IEEE Trans. Commun. 51(11), 1927-1938 (2003)

7. TM Cover, A El Gamal, Capacity theorems for the relay channel. IEEE Trans. Inf. Theory. 25(5), 572-584 (1979)

8. A El Gamal, M Mohseni, S Zahedi, Bounds on capacity and minimum energy-per-bit for AWGN relay channels. IEEE Trans. Inf. Theory. 52(4), 1545-1561 (2006)

9. JN Laneman, Cooperative Diversity in Wireless networks: Algorithms and Architectures. (Ph.D. dissertation, Massachusetts Institute of Technology, Cambridge, MA, 2002)

10. M Souryal, H You, in WCNC 2008. Quantize-and-Forward Relaying with M-ary Phase Shift Keying (IEEE Wireless Communications and Networking Conference Las Vegas, United, States, 31 Mar. -3 Apr 2008), pp. 42-47

11. JN Laneman, DNC Tse, GW Wornell, Cooperative diversity in wireless networks: efficient protocols and outage behavior. IEEE Trans. Inf. Theory. 50(12), 3062-3080 (2004)

12. TE Hunter, S Sanayei, A Nosratinia, Outage analysis of coded cooperation. IEEE Trans. Inf. Theory. 52(2), 375-391 (2006)

13. YW Hong, WJ Huang, FH Chiu, CCJ Kuo, Cooperative communications in resource-constrained wireless networks. IEEE Signal Process. Mag. 24(3), 47-57 (2007)

14. I Avram, N Aerts, D Duyck, M Moeneclaey, A Novel Quantize-and-Forward Cooperative System: Channel Estimation and M-PSK Detection Performance. EURASIP J. Wirel. Commun. Netw. Article, ID 415438, 11 pages (2010)
15. I Avram, N Aerts, M Moeneclaey, A Novel Quantize-and-Forward Cooperative System: Channel Parameter Estimation Techniques. (Future Network \& Mobile Summit 2010, Florence, Italy, 16-18 Jun 2010)

16. I Avram, N Aerts, H Bruneel, M Moeneclaey, Quantize and forward cooperative communication: channel parameter estimation. IEEE Trans. Wireless Commun. 11(3), 1167-1179 (2012)

17. CR Rao, Information and the accuracy attainable in the estimation of statistical parameters. Bulletin of Cal. Math. Soc. 37(3), 81-91 (1945)

18. S Zhang, F Gao, J Li, M Sheng, Online and offline Bayesian Cramer-Rao bounds for time-varying channel estimation in one-way relay networks, IEEE Trans. Signal Process. 63(8), 1977-1992 (2015)

19. H Mehrpouyan, SD Blostein, Bounds and algorithms for multiple frequency offset estimation in cooperative networks. IEEE Trans. Wireless Commun. 10(4), 1300-1311 (2011)

20. Z Jiang, H Wang, Z Ding, A Bayesian algorithm for joint symbol timing synchronization and channel estimation in two-way relay networks. IEEE Trans. Wireless Commun. 61(10), 4271-4283 (2013)

21. S Abdallah, IN Psaromiligkos, Partially-blind estimation of reciprocal channels for af two-way relay networks employing M-PSK modulation. IEEE Trans. Wireless Commun. 11(5), 1649-1654 (2012)

22. S Abdallah, IN Psaromiligkos, Blind channel estimation for amplifyand-forward two-way relay networks employing M-PSK modulation. IEEE Trans. Signal Process. 60(7), 3604-3615 (2012)

23. S Zhang, F Gao, C-X Pei, Optimal training design for individual channel estimation in two-way relay networks. IEEE Trans. Signal Process. 60(9), 4987-4991 (2012)

24. AD Andrea, U Mengali, R Reggiannini, The modified Cramer-Rao bound and its application to synchronization problems. IEEE Trans. Commun. 42 1391-1399 (1994)

25. M Moeneclaey, On the True and the modified Cramer-Rao bounds for the estimation of a scalar parameter in the presence of nuisance parameters. IEEE Trans. Commun. 46(11), 1536-1544 (1998)

26. I Avram, M Moeneclaey, in WCNC 2013. The Modified Cramer-Rao Bound for Channel Estimation in Quantize-and-Forward Cooperative Systems (IEEE Wireless Communications and Networking Conference (WCNC) Shanghai, China, 7-10 Apr 2013), pp. 2862-2867

27. M Woodbury, Inverting modified matrices. (Memorandum Rept. 42, Statistical, Research Group, Princeton University, Princeton, NJ, 1950)

28. JM Hammersley, DC Handscomb, Monte Carlo Methods. (Methuen \& Co. London, and John Wiley \& Sons, New York, 1964)

29. S Lin, D Costello, Error Control Coding. (Pearson Education Inc., second edition, 2004)

\section{Submit your manuscript to a SpringerOpen ${ }^{\circ}$ journal and benefit from:}

- Convenient online submission

- Rigorous peer review

- Immediate publication on acceptance

- Open access: articles freely available online

- High visibility within the field

- Retaining the copyright to your article

Submit your next manuscript at $\gg$ springeropen.com 